Publ. RIMS, Kyoto Univ.

13 (1977), 173-192

\title{
Relative Entropy for States of von Neumann Algebras II
}

By

\author{
Huzihiro ARAKI*
}

\begin{abstract}
Earlier definition of the relative entropy of two faithful normal positive linear functionals of a von Neumann algebra is generalized to non-faithful functionals. Basic properties of the relative entropy are proved for this generalization.
\end{abstract}

\section{§. Introduction}

For two faithful normal positive linear functionals $\phi$ and $\psi$ of a von Neumann algebra $M$, the relative entropy $S(\psi \mid \phi)$ is defined and its properties are proved in an earlier paper [1].

When $M$ is a finite dimensional factor, it is given by

$$
S(\psi \mid \phi)=\phi\left(\log \rho_{\phi}-\log \rho_{\psi}\right)
$$

where $\rho_{\phi}$ and $\rho_{\psi}$ are density matrices for $\phi$ and $\psi$. If $\psi$ and $\phi$ are faithful, $\rho_{\phi}$ and $\rho_{\phi,}$ are strictly positive and (1.1) clearly makes sense. However the first term of (1.1) always makes sense (under the convention $\lambda \log \lambda=0$ for $\lambda=0$ ) and the second term is either finite or infinite. Therefore (1.1) can be given an unambiguous finite or positive infinite value for every $\phi$ and $\psi$.

We shall make corresponding generalization for an arbitrary von Neumann algebra $M$ and any normal positive linear functionals $\psi$ and $\phi$. We shall also define the relative entropy of two positive linear functionals of a $C^{*}$-algebra 9 and give an alternative proof of a result of [2]. For the latter case, we relate the conditional entropy introduced in [3] with our relative entropy.

Received September 10, 1976.

* ZiF, Universität Pielefeld, BRD, Permanent address: Research Institute for Mathematical Sciences, Kyoto University, Kyoto 606, JAPAN 
The relative entropy for non-faithful functionals will be shown to satisfy all properties proved for faithful functionals in [1]. Some of these properties will be applied to a discussion of local thermodynamical stability in [3].

For simplicity, we shall assume that $M$ has a faithful normal state although many of the results are independent of this assumption.

\section{§ 2. Relative Modular Operator}

Let $\Phi$ and $\Psi$ be vectors in a natural positive cone $V([4],[5],[6])$ for a von Neumann algebra $M$ on a Hilbert space $H$ and let $\phi$ and $\psi$ be the corresponding normal positive linear functionals of $M$. Let $s^{R}(\Omega)$ denote the $R$-support of a vector $\Omega$, where $R$ is a von Neumann algebra.

Definition 2. 1. Operators $S_{\emptyset, \Psi}$ and $F_{\oplus, \Psi}$ with their domains

$$
\begin{aligned}
& D\left(S_{\mathscr{\theta}, \Psi}\right)=M \Psi+\left(\mathbb{1}-s^{M^{\prime}}(\Psi)\right) H, \\
& D\left(F_{\mathscr{Q}, \Psi}\right)=M^{\prime} \Psi+\left(\mathbb{1}-s^{M}(\Psi)\right) H,
\end{aligned}
$$

are defined by

$$
\begin{aligned}
& S_{\Phi, \Psi}\{x \Psi+\Omega\}=s^{M}(\Psi) x^{*} \Phi, \\
& F_{\mathscr{\Phi}, \Psi}\left\{x^{\prime} \Psi+\Omega^{\prime}\right\}=s^{M \prime}(\Psi) x^{*} \Phi,
\end{aligned}
$$

where $x \in M, x^{\prime} \in M^{\prime}, s^{M \prime^{\prime}}(\Psi) \Omega=0, s^{M}(\Psi) \Omega^{\prime}=0$.

Lemma 2.2. $S_{\emptyset, \Psi}$ and $F_{\emptyset, \Psi}$ are closable antilinear operators.

Proof: If $x_{1} \Psi+\Omega_{1}=x_{2} \Psi+\Omega_{2} \quad$ for $\quad x_{1}, x_{2} \in M \quad$ and $\quad \Omega_{1}, \Omega_{2} \in(\mathbb{1}-$ $\left.s^{M N^{\prime}}(\Psi)\right) H$, then $\Omega_{1}=\Omega_{2}$ and $\left(x_{1}-x_{2}\right) s^{M}(\Psi)=\mathbf{0}$, so that $s^{M}(\Psi) x_{1} * \Phi=$ $s^{M}(\Psi) x_{2}{ }^{*} \Phi$. This shows that $S_{\mathscr{\Phi}, \Psi}$ is well-defined. Then it is clearly antilinear. Similarly $F_{\mathscr{Q}, \Psi}$ is an antilinear operator.

Let $x \in M, x^{\prime} \in M^{\prime}, s^{H^{\prime}}(\Psi) \Omega=s^{M}(\Psi) \Omega^{\prime}=0$. Then

$$
\begin{gathered}
\left(S_{\emptyset, \Psi}\{x \Psi+\Omega\},\left\{x^{\prime} \Psi+\Omega^{\prime}\right\}\right)=\left(x^{*} \Phi, x^{\prime} \Psi\right) \\
=\left(F_{\emptyset, \Psi}\left\{x^{\prime} \Psi+\Omega\right\},\{x \Psi+\Omega\}\right) .
\end{gathered}
$$

Since $S_{\mathscr{\Phi} \Psi}$ and $F_{\mathscr{Q}, \Psi}$ have dense domains, this shows the closability of 
$S_{\emptyset, \Psi}$ and $F_{\emptyset, \Psi}$.

Definition 2. 3. The relative modular operator $\Delta_{\mathscr{G}, \Psi}$ is defined by

$$
\Delta_{\mathscr{G}, \Psi}=\left(S_{\mathscr{\varphi}, \Psi}\right) * \bar{S}_{\emptyset, \Psi}
$$

where the bar denotes the closure.

We denote by $J$ the modular conjugation operator associated with the natural positive cone $\mathrm{V}$.

\section{Theorem 2.4.}

(1) The kernel of $\Delta_{\mathscr{\Phi} . \Psi}$ is $\mathbb{1}-s^{M^{\prime}}(\Psi) s^{M}(\Phi)$.

(2) The following formulas hold, where the bar denotes the closure.

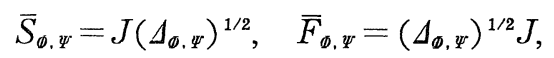

(3) If $s^{M}\left(\Phi_{1}\right) \perp s^{M}\left(\Phi_{2}\right)$, then

$$
\Delta_{\mathscr{\omega}_{1}+\oplus_{2}, \Psi}=\Delta_{\mathscr{\Phi}_{1}, \Psi}+\Delta_{\Phi_{2}, \Psi} .
$$

Proof:

(1) and (2): First we prove Theorem for the special case $\Phi=\Psi$. The domain of $S_{\mathbb{Y}, \Psi^{r}}$ is split into a direct sum of 3 parts:

$$
D\left(S_{\Psi, \psi}\right)=s^{M}(\Psi) M \Psi+\left(\mathbb{1}-s^{M}(\Psi)\right) M \Psi+\left(\mathbb{1}-s^{M{ }^{\prime}}(\Psi)\right) H .
$$

Accordingly, we split $S_{\Psi, Y}$ as a direct sum

$$
S_{Y, \Psi}=\widehat{S}_{Y, \Psi} \oplus \oplus \oplus \mathbb{O}
$$

where $S_{\Psi, y}$ is the operator on $s^{M}(\Psi) s^{M \prime}(\Psi) H$ defined by

$$
\widehat{S}_{\Psi, \Psi} x \Psi=x^{*} \Psi, \quad x \in s^{M}(\Psi) M s^{M}(\Psi)
$$

and the splitting of the Hilbert space is

$$
H=s^{M}(\Psi) s^{M^{\prime}}(\Psi) H \oplus\left(\mathbb{1}-s^{M}(\Psi)\right) s^{M^{\prime}}(\Psi) H \oplus\left(\mathbb{1}-s^{M^{\prime}}(\Psi)\right) H .
$$

Since $\Psi$ is cyclic and separating relative to $s^{M}(\Psi) M s^{M}(\Psi)$ in the subspace $s^{M}(\Psi) s^{M M^{\prime}}(\Psi) H$

$$
\Delta_{\Psi, Y}=\tilde{\Delta}_{\Psi, Y} \oplus \mathbf{O} \oplus \mathbf{0}
$$


where $\tilde{\Delta}_{\Psi, \Psi}$ is the modular operator of $\Psi$ relative to $s^{M}(\Psi) M s^{M}(\Psi)$. Since ${ }^{1)} s^{M^{\prime}}(\Psi)=J s^{M}(\Psi) J, J$ commutes with $s^{M}(\Psi) s^{M^{\prime}}(\Psi)$ and hence leaves $s^{M}(\Psi) s^{H^{\prime}}(\Psi) H$ invariant. The restriction of $J$ to this subspace is the modular conjugation operator for $\Psi$, as can be checked by the characterization of $J$ given in [4]. Therefore the known property of the modular operator for a cyclic and separating vector implies (1) and (2) for the case $\Psi=\Phi$.

To prove (1) and (2) for the general case, we use the $2 \times 2$ matrix method of Connes [7]. Let $\widetilde{M}=M \otimes M_{2}$ with $M_{2}$ a type $I_{2}$ factor on a 4-dimensional space $K$, let $u_{i j}$ be a matrix unit of $M_{2}$, let $e_{i j}$ be an orthonormal basis of $K$ satisfying $u_{i j} e_{k l}=\delta_{j k} e_{i l}$, let $J_{K}$ be the modular conjugation operator of $e_{11}+e_{22}$ (i.e. $J_{K} e_{i j}=e_{j i}$ ), and let

$$
\Omega=\sum \Omega_{j} \otimes e_{j j}
$$

with $\Omega_{1}=\Psi$ and $\Omega_{2}=\Phi$. From definition, we obtain

$$
\begin{aligned}
& s^{\widetilde{H}}(\Omega)=\sum s^{M}\left(\Omega_{j}\right) \otimes u_{j j}, \\
& s^{\widetilde{M}^{\prime}}(\Omega)=\sum s^{H^{\prime}}\left(\Omega_{j}\right) \otimes J_{K} u_{j j} J_{K}, \\
& \left(\mathbf{1} \otimes u_{i i}\right) S_{\Omega . \Omega}\left(\mathbf{1} \otimes u_{j j}\right)=S_{\Omega_{j, \Omega_{i}}} \otimes u_{i i} J_{K} u_{j j} .
\end{aligned}
$$

Since the modular conjugation operator $J$ for the natural positive cone of $M$ containing $V \otimes\left(e_{1}+e_{2}\right)$ is given by $J \otimes J_{K}$, we obtain

$$
\Delta_{\Omega, \Omega}\left(\mathbb{1} \otimes J_{K} u_{i i} J_{K} u_{j j}\right)=\Delta_{\Omega_{j, \Omega_{i}}} \otimes J_{K} u_{i i} J_{K} u_{j j} .
$$

Hence (1) and (2) proved above for $\Delta_{\Omega, \Omega}$ imply the same for $\Delta_{\mathscr{Q}, \Psi}$ and $\Delta_{\Psi, \mathscr{\varphi}}$.

(3) If $s^{M}\left(\Phi_{j}\right)$ is mutually orthogonal for $j=1,2$, then the same holds for $s^{M L^{\prime}}\left(\Phi_{j}\right)=J s^{M}\left(\Phi_{j}\right) J$. By (1) and (2), the range projection of $S_{\mathscr{O}_{j} T}$ is

$$
J s^{M{ }^{\prime}}(\Psi) s^{M}\left(\Phi_{j}\right) J=s^{M}(\Psi) s^{M^{\prime}}\left(\Phi_{j}\right)
$$

and is mutually orthogonal for $j=1,2$. The same holds for the corange projection. From definition we obtain

$$
S_{\mathscr{\omega}_{1}+\oplus_{2}, \Psi}=S_{\mathscr{\omega}_{1}, \psi^{r}}+S_{\emptyset_{2}, \Psi} .
$$

Hence we obtain (2.6).

Q.E.D.

\footnotetext{
1) This follows from $J \Psi=\Psi$.
} 


\section{$\S 3$. Relative Entropy for States of von Neumann Algebras}

Let $M, \Psi, \Phi, \psi$ and $\phi$ be as in the previous section. Let $E_{\lambda}{ }^{\Phi, \Psi}$ denote the spectral projections of $\Delta_{\mathscr{Q}, \Psi}, s(\omega)$ denote the support of the positive linear functional $\omega$.

Definition 3. 1. For $\phi \neq 0$, the relative entropy $S(\psi / \phi)$ is defined by

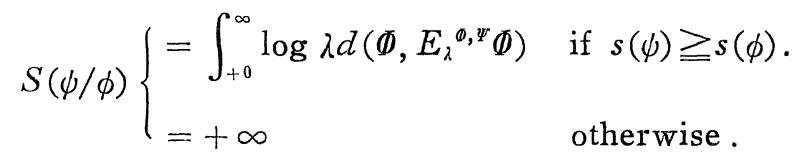

Lemma 3.2. $S(\psi / \phi)$ is well defined, takes finite value or $+\infty$ and satisfies

$$
S(\psi / \phi) \geqq-\phi(\mathbb{1}) \log \{\psi(s(\phi)) / \phi(\mathbb{1})\}
$$

Proof: First consider the case $s(\psi) \geqq s(\phi)$. Since $s^{M}(\Psi)=s(\psi)$ $\geqq s(\phi)=s^{M}(\Phi)$, we have $S_{\emptyset, \Psi} \Psi=\Phi$.

Since $J \Phi=\Phi$, we have $\left(d_{\mathscr{Q}, \Psi}\right)^{1 / 2} \Psi=\Phi$. Hence

$$
\begin{aligned}
\int_{+0}^{\infty} \lambda^{-1} \mathrm{~d}\left(\Phi, E_{\lambda}^{\Phi, \Psi} \Phi\right) & =\left(\Psi,\left(\mathbb{1}-E_{+0}^{\oplus, \Psi}\right) \Psi\right) \\
& =\left(\Psi, s^{M I^{\prime}}(\Psi) s^{M}(\Phi) \Psi\right) \\
& =(\Psi, s(\phi) \Psi)=\psi(s(\phi)) \leqq 1
\end{aligned}
$$

This implies that the integral defining $S(\psi / \phi)$ converges at the lower end. Hence it is well defined and takes either finite value or $+\infty$.

Since $s(\psi) \geqq s(\phi)$ implies

$$
\int_{-0}^{\infty} \mathrm{d}\left(\Phi, E_{l}^{\Phi, \Psi} \Phi\right)=\left(\Phi, s^{M \prime}(\Psi) s^{M}(\Phi) \Phi\right)=\phi(\mathbb{1}),
$$

$\mathrm{d}\left(\Phi, E_{\mathrm{\lambda}}^{\oplus, \Psi} \Phi\right) / \phi(\mathbf{1})$ is a probability measure on $(0,+\infty)$. By the concavity of the logarithm, we obtain

$$
\begin{aligned}
S(\psi / \phi) & =-\phi(\mathbb{1}) \int_{+0}^{\infty} \log \left(\lambda^{-1}\right) \mathrm{d}\left(\Phi, E_{\lambda}^{\Phi, \Psi} \Phi\right) / \phi(\mathbb{1}) \\
& \geqq-\phi(\mathbb{1}) \log \left\{\int_{+0}^{\infty} \lambda^{-1} \mathrm{~d}\left(\Phi, E_{\lambda}^{\Phi, \Psi} \Phi\right) / \phi(\mathbb{1})\right\}
\end{aligned}
$$




$$
=-\phi(\mathbb{1}) \log \{\psi(s(\phi)) / \phi(\mathbb{1})\} .
$$

The statement of Lemma holds trivially for the case where $s(\psi) \geqq s(\phi)$ does not hold.

Remark 3.3. The definition of $S(\psi / \phi)$ uses the (unique) vector representatives $\Psi$ and $\Phi$ in a natural positive cone V. The value $S(\psi / \phi)$, however does not depend on the choice of the natural positive cone $\mathrm{V}$ because of the following reason. If $\mathrm{V}^{\prime}$ is another natural positive cone, then there exists a unitary $w^{\prime} \in M^{\prime}$ such that $V^{\prime}=w^{\prime} V . \quad \Psi^{\prime}=w^{\prime} \Psi$ and $\Phi^{\prime}=w^{\prime} \bar{\Phi}$ are representative vectors of $\psi$ and $\phi$ in $V^{\prime}$. We then obtain $\Delta_{\Phi^{\prime}, \Psi^{\prime}}=w^{\prime} \Delta_{\mathscr{\Phi}, \Psi}\left(w^{\prime}\right)^{*}$ and hence $S(\psi / \phi)$ is unchanged.

Remark 3. 4. By Theorem 2.4 (2), we have

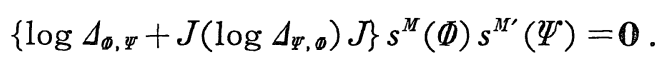

Hence, for the case $s(\psi) \geqq s(\phi)$, we obtain the following expression ([1]):

$$
S(\psi / \phi)=-\left(\Phi, \log \Delta_{\Psi, \Phi} \Phi\right)
$$

Remark 3.5. If $s(\psi)=s(\phi)$, then $\Delta_{\mathscr{E}, \Psi}$ is $\mathbf{0}$ on $(\mathbb{1}-s(\psi) J s(\psi) J) H$ and coincides with the relative modular operator for $s(\psi) M s(\psi)$ on the space $s(\psi) J s(\psi) J H$, where $\Phi$ and $\Psi$ are cyclic and separating for $s(\psi) M s(\psi)$. Hence $S(\psi / \phi)$ in this case is the same as the relative entropy of two faithful normal positive linear functionals $\psi$ and $\phi$ of $s(\psi) M s(\psi)$.

\section{Theorem 3.6.}

(1) If $\psi(\mathbb{1})=\phi(\mathbb{1})>0$, then $S(\psi / \phi) \geqq 0$. The equality $S(\psi / \phi)=0$ holds if and only if $\psi=\phi$.

(2) If $s\left(\phi_{1}\right) \perp s\left(\phi_{2}\right)$, then

$$
S\left(\psi / \phi_{1}\right)+S\left(\psi / \phi_{2}\right)=S\left(\psi / \phi_{1}+\phi_{2}\right) .
$$

(3) For $\lambda_{1}, \lambda_{2}>0$,

$$
S\left(\lambda_{1} \psi / \lambda_{2} \phi\right)=\lambda_{2} S(\psi / \phi)-\lambda_{2} \phi(\mathbf{1}) \log \left(\lambda_{1} / \lambda_{2}\right) .
$$

(4) If $\psi_{1} \geqq \psi_{2}$, then 


$$
S\left(\psi_{1} / \phi\right) \leqq S\left(\psi_{2} / \phi\right)
$$

Proof.

(1) Since $\psi(s(\phi)) \leqq \psi(\mathbb{1})$, the assumption $\psi(\mathbb{1})=\phi(\mathbb{1})$ and (3.1) imply $S(\psi / \phi) \geqq 0$. Furthermore, the equality $S(\psi / \phi)=0$ holds only if $s(\psi) \geqq s(\phi)$ and $\psi(s(\phi))=\psi(\mathbf{1})$. We then have $s(\phi)=s(\psi)$; hence Remark 3.5 and the strict positivity of $S(\psi / \phi)$ for faithful $\psi$ and $\phi$ ([1]) imply $\phi=\psi$ also in the present case. Conversely $\phi=\psi$ implies $S(\psi / \phi)=0$.

(2) (3. 4) follows from (2.6) and Definition 3.1.

(3) The vector representatives for $\lambda_{1} \psi$ and $\lambda_{2} \phi$ differs from those for $\psi$ and $\phi$ by factors $\left(\lambda_{1}\right)^{1 / 2}$ and $\left(\lambda_{2}\right)^{1 / 2}$ respectively. Hence this induces

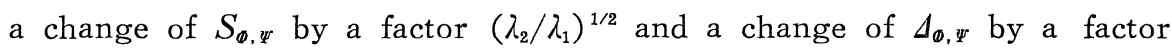
$\left(\lambda_{2} / \lambda_{1}\right)$. The latter proves (3.5).

(4) If $s\left(\psi_{2}\right) \geqq s(\phi)$ does not hold, then (3.6) is trivially true. Hence we assume $s\left(\psi_{2}\right) \geqq s(\phi)$. Since $\psi_{1} \geqq \psi_{2}$ implies $s\left(\psi_{1}\right) \geqq s\left(\psi_{2}\right)$, we also have $s\left(\psi_{1}\right) \geqq s(\phi)$. The following proof is then the same as that for the case of faithful $\psi$ 's and $\phi$ :

Denoting representative vectors of $\psi_{1}, \psi_{2}$ and $\phi$ in the natural positive cone by $\Psi_{1}, \Psi_{2}$ and $\Phi$, respectively, we obtain

$$
\begin{aligned}
& \left\|\left(\Delta_{\Psi_{1}, \oplus}\right)^{1 / 2} x \Phi\right\|^{2}=\left\|S_{\psi_{1}, \varpi} x \Phi\right\|^{2}=\left\|s(\phi) x^{*} \Psi_{1}\right\|^{2} \\
& \quad=\psi_{1}\left(x s(\phi) x^{*}\right) \geqq \psi_{2}\left(x s(\phi) x^{*}\right)=\left\|\left(\Delta_{\Psi_{2}, \oplus}\right)^{1 / 2} x \Phi\right\|^{2},
\end{aligned}
$$

for all $x \in M$. Since both $\left(\Delta_{\Psi_{j}, \emptyset}\right)^{1 / 2}$ vanish on $\left(s^{W^{\prime}}(\Phi) H\right)^{\perp}$ and since $M \Phi+\left(\mathbf{1}-s^{M^{\prime}}(\Phi)\right) H$ is the core of $\left(\Delta_{\Psi_{1}, \emptyset}\right)^{1 / 2}$, it follows that the domain of $\left(\Delta_{\Psi_{1}, \varphi}\right)^{1 / 2}$ is contained in the domain of $\left(\Delta_{\Psi_{2}, \Theta}\right)^{1 / 2}$ and for all $\Omega$ in the domain of $\left(\Delta_{\Psi_{1}, \oplus}\right)^{1 / 2}$

$$
\left\|\left(\Delta_{Y_{1}, \oplus}\right)^{1 / 2} \Omega\right\| \geqq\left\|\left(\Delta_{\Psi_{2}, \oplus}\right)^{1 / 2} \Omega\right\| .
$$

Hence

$$
\left\|\left(\Delta_{\Psi_{1}, \varpi}+r\right)^{1 / 2} \Omega\right\|^{2} \geqq\left\|\left(\Delta_{\Psi_{2}, \oplus}+r\right)^{1 / 2} \Omega\right\|^{2}
$$

for all such $\Omega$ and $r>0$. Taking $\Omega=\left(\Delta_{T_{1}, \oplus}+r\right)^{-1 / 2} \Omega^{\prime}$ with an arbitrary $\Omega^{\prime}$, we find 


$$
\left\|\left(\Delta_{\Psi_{2}, \oplus}+r\right)^{1 / 2}\left(\Delta_{\Psi_{1}, \oplus}+r\right)^{-1 / 2}\right\| \leqq 1 .
$$

Taking adjoint operator acting on $\Omega=\left(\Delta_{\Psi_{2}, \emptyset}+r\right)^{-1 / 2} \Omega^{\prime}$ with an arbitrary $\Omega^{\prime}$, we find

$$
\left\|\left(\Delta_{\Psi_{1}, \oplus}+r\right)^{-1 / 2} \Omega^{\prime}\right\|^{2} \leqq\left\|\left(\Delta_{\Psi_{2}, \emptyset}+r\right)^{-1 / 2} \Omega^{\prime}\right\|^{2}
$$

and hence

$$
\left(\Delta_{\Psi_{1}, \oplus}+r\right)^{-1} \leqq\left(d_{\Psi_{2}, \odot}+r\right)^{-1}
$$

By (3.3) we have

$$
\text { (3. 8) } \begin{aligned}
S\left(\psi_{j} / \phi\right) & =-\int_{0}^{\infty}\left\{\int_{0}^{\infty}\left[(1+r)^{-1}-(\lambda+r)^{-1}\right] \mathrm{d} r\right\} \mathrm{d}\left(\Phi, E_{\lambda}^{\Psi_{j}, \Phi} \Phi\right) \\
& =\int_{0}^{\infty}\left(\Phi,\left[\left(r+\Delta_{\Psi_{j}, \boldsymbol{\varphi}}\right)^{-1}-(1+r)^{-1}\right] \Phi\right) d r
\end{aligned}
$$

where $E_{\lambda}^{\Psi_{j, \oplus}}$ is the spectral projection of $\Delta_{\Psi_{j, \oplus}}$ and the interchange of $r$ - and $\lambda$-integrations are allowed because the double integral is definite in the Lebesque sense (finite or $+\infty$ ) due to

$$
\int_{0}^{\infty} \lambda \mathrm{d}\left(\Phi, E_{\lambda}{ }^{\Psi_{j, \Phi}} \Phi\right)=\left\|\left(\Delta_{\Psi_{j,}}\right)^{1 / 2} \Phi\right\|^{2}=\left\|s(\phi) \Psi_{j}\right\|^{2}<\infty .
$$

The equations (3.8) and (3.7) imply (3.6).

The following Theorem describes the continuity property of $S(\psi / \phi)$ as a function of $\psi$ and $\phi$. (It is the same as the case of faithful $\psi$ and $\phi$.)

\section{Theorem 3. 7.}

Assume that $\lim \left\|\phi_{\alpha}-\phi\right\|=\lim \left\|\psi_{\alpha}-\psi\right\|=0$.

(1) $\lim \inf S\left(\psi_{\alpha} / \phi_{\alpha}\right) \geqq S(\psi / \phi)$ (the lower semicontinuity).

(2) If $\lambda \psi_{\alpha} \geqq \phi_{\alpha}$ for a fixed $\lambda>0$, then

$$
\lim S\left(\psi_{\alpha} / \phi_{\alpha}\right)=S(\psi / \phi) .
$$

(3) If $\psi_{\alpha}$ is monotone decreasing, then

$$
\lim S\left(\psi_{\alpha} / \phi\right)=S(\psi / \phi) .
$$

We shall give proof of this Theorem in the next section. Using this theorem in an approximation argument, we obtain the next theorem from the same theorem ([1]) for faithful functionals. 


\section{Theorem 3. 8.}

(1) $S(\psi / \phi)$ is jointly convex in $\psi$ and $\phi$.

(2) Let $N$ be a von Neumann subalgebra of $M$ and $E_{N} \omega$ denotes the restriction of a functional (1) to $N$. Then

$$
S\left(E_{N} \psi / E_{N} \phi\right) \leqq S(\psi / \phi)
$$

if $N$ is any one of the following type:

( $\alpha) \quad N=\mathfrak{X}^{\prime} \cap M$ for a finite dimensional abelian *-subalgebra $\mathfrak{I}$ of $M$.

(ß) $\quad M=N \otimes N_{1}$.

$(\gamma) \quad N$ is approximately finite.

\section{Proof.}

(1) We have to prove the following

$$
S\left(\sum_{j=1}^{n} \lambda_{j} \psi_{j} / \sum_{j=1}^{n} \lambda_{j} \phi_{j}\right) \leqq \sum_{j=1}^{n} \lambda_{j} S\left(\psi_{j} / \phi_{j}\right)
$$

for $\lambda_{j}>0, \sum \lambda_{j}=1$. Let $\psi=\sum \lambda_{j} \psi_{j}, \phi=\sum \lambda_{j} \phi_{j}, \omega=\psi+\phi$. By Remark 3. 5,

$$
S(\psi+\varepsilon \omega / \phi+\eta \omega) \leqq \sum_{j=1}^{n} \lambda_{j} S\left(\psi_{j}+\varepsilon \omega / \phi_{j}+\eta \omega\right)
$$

follows from the convexity of $S\left(\psi_{0} / \phi_{0}\right)$ for faithful $\psi_{0}$ and $\phi_{0}$. We first take the limit $\eta \rightarrow+0$ using Theorem 3.7 (2) and then take the limit $\varepsilon \rightarrow+0$ using Theorem 3.7 (3) to obtain (3.10).

(2) Let $\omega_{0}$ be a faithful normal state of $M$ and let $\omega=\omega_{0}+\phi+\psi$. Then

$$
\left.S\left(E_{N}(\psi+\varepsilon \omega) / E_{N}(\phi+\eta \omega)\right) \leqq S(\psi+\varepsilon \omega) / \phi+\eta \omega\right) .
$$

Again Theorem 3.7 (2) and (3) yield (3.9).

Q.E.D.

The following Theorem describe some continuity property of $S\left(E_{N} \psi /\right.$ $\left.E_{N} \phi\right)$ on $N$.

Theorem 3.9. Let $N_{a}$ be monotone increasing net of von Neumann subalgebras of $M$ generating $M$.

(1) $\lim \inf S\left(E_{N_{\alpha}} \psi / E_{N_{a}} \phi\right) \geqq S(\psi / \phi)$.

(2) If $N_{a}$ is an AF algebra for all $\alpha$, then 
$\lim S\left(E_{N_{\alpha}} \psi / E_{N_{\alpha}} \phi\right)=S(\psi / \phi)$

Proof of (1) and (2) will be given in the next section. (2) follows from (1) and Theorem 3.8 (2) ( $\gamma$ ).

Let $\psi$ be a faithful normal positive linear functional of $M$ corresponding to a cyclic and separating vector $\Psi$ and $h=h^{*} \in M$. Let $\Psi(h)$ denote the perturbed vector defined by (4.1) in [8]. Let $\psi^{h}$ denote the perturbed state defined by

$$
\psi^{h}(x)=(\Psi(h), x \Psi(h)), \quad x \in M
$$

\section{Theorem 3. 10 .}

$$
\begin{aligned}
& S\left(\psi^{h} / \phi\right)=-\phi(h)+S(\psi / \phi), \\
& S\left(\phi / \psi^{h}\right)=\psi^{h}(h)+S\left(\phi^{h} / \psi^{h}\right) .
\end{aligned}
$$

\section{$\S 4$. Some Continuity Properties}

We first prove some continuity properties of the relative modular operators.

Lemma 4. 1. If $\lim \left\|\phi_{\alpha}-\phi\right\|=\lim \left\|\psi_{\alpha}-\psi\right\|=0$, then

$$
\lim \left(r+\left(\Delta_{\Phi_{\alpha}, \Psi_{\alpha}}\right)^{1 / 2}\right)^{-1} s^{M H^{\prime}}(\Psi)=\left(r+\left(\Delta_{\mathscr{\oplus}, \Psi}\right)^{1 / 2}\right)^{-1} s^{M H^{\prime}}(\Psi)
$$

for $r>0$ and the convergence is uniform in $r$ if $r$ is restricted to any compact subset of $(0, \infty)$, where $\Phi_{\alpha}, \Psi_{a}, \Phi$ and $\Psi$ are the representative vectors of $\phi_{\alpha}, \psi_{\alpha}$, $\phi$ and $\psi$ in the positive natural cone, respectively.

Proof. The condition $\lim \left\|\phi_{\alpha}-\phi\right\|=\lim \left\|\psi_{\alpha}-\psi^{\prime}\right\|=0$ implies (Theorem $4(8)$ in $[4])$

$$
\lim \left\|\Phi_{\alpha}-\Phi\right\|=\lim \left\|\Psi_{\alpha}-\Psi\right\|=0
$$

For $x^{\prime} \in M I^{\prime}$, we have

$$
\begin{aligned}
& \lim \left\|s^{M}\left(\Psi_{\alpha}\right) x^{\prime} \Psi-x^{\prime} \Psi\right\| \\
& \quad=\lim \left\|s^{M}\left(\Psi_{\alpha}\right) x^{\prime}\left(\Psi-\Psi_{\alpha}\right)+x^{\prime}\left(\Psi_{\alpha}-\Psi\right)\right\|=0 .
\end{aligned}
$$


Hence

$$
\lim s^{M}\left(\Psi_{\alpha}\right) s^{M}(\Psi)=s^{M}(\Psi) .
$$

For $x \in M s^{M}(\Psi)$, we have

$$
\begin{aligned}
& \left\|\left(\Delta_{\Phi_{\alpha}, \Psi_{\alpha}}\right)^{1 / 2} x \Psi_{a}-\left(\Delta_{\varpi, \Psi}\right)^{1 / 2} x \Psi\right\| \\
& \quad=\left\|J\left(\Delta_{\Phi_{\alpha}, \Psi_{\alpha}}\right)^{1 / 2} x \Psi_{\alpha}-J\left(\Delta_{\varpi, \Psi}\right)^{1 / 2} x \Psi\right\| \\
& \quad=\left\|s^{M}\left(\Psi_{\alpha}\right) x^{*} \Phi_{\alpha}-x^{*} \Phi\right\| \\
& \quad \leqq\left\|\left(s^{M I}\left(\Psi_{\alpha}\right) s^{M}(\Psi)-s^{M}(\Psi)\right) x^{*} \Phi\right\|+\left\|s^{M}\left(\Psi_{\alpha}\right) x^{*}\left(\Phi_{\alpha}-\Phi\right)\right\| \rightarrow 0 .
\end{aligned}
$$

Hence

$$
\begin{aligned}
& \left\|\left\{\left[\mathbb{1}+\left(\Delta_{\Phi_{\alpha}, \Psi_{\alpha}}\right)^{1 / 2}\right]^{-1}-\left[\mathbb{1}+\left(\Delta_{\mathscr{\oplus}, \Psi}\right)^{1 / 2}\right]^{-1}\right\}\left[\mathbb{1}+\left(\Delta_{\mathscr{\varphi}, \Psi}\right)^{1 / 2}\right] x \Psi\right\| \\
& =\|\left[\mathbf{1}+\left(\Delta_{\mathscr{Q}_{\alpha}, \Psi_{\alpha}}\right)^{1 / 2}\right]^{-1} \\
& \times\left\{x\left(\Psi-\Psi_{\alpha}\right)+\left[\left(\Delta_{\varpi, \Psi}\right)^{1 / 2} x \Psi-\left(\Delta_{\oplus_{\alpha}, \Psi_{\alpha}}\right)^{1 / 2} x \Psi_{\alpha}\right]\right\}+x\left(\Psi_{\alpha}-\Psi\right) \| \\
& \leqq 2\left\|x\left(\Psi_{\alpha}-\Psi\right)\right\|+\left\|\left(\Delta_{\varpi_{\alpha}, \Psi_{\alpha}}\right)^{1 / 2} x \Psi_{\alpha}-\left(\Delta_{\varpi, \Psi}\right)^{1 / 2} x \Psi\right\| \rightarrow 0 \text {. }
\end{aligned}
$$

Since $M s^{M}(\Psi) \Psi+\left(\mathbb{1}-s^{M^{\prime}}(\Psi)\right) H$ is a core for $\left(\Delta_{\mathscr{Q}, \Psi}\right)^{1 / 2}$, the vectors

$$
\left(\mathbb{1}+\left(\Delta_{\mathscr{\emptyset}, \Psi}\right)^{1 / 2}\right) x \Psi, \quad x \in M s^{M I}(\Psi)
$$

are dense in $s^{M^{\prime}}(\Psi) H$. Since

$$
\left\|\left[\mathbb{1}+\left(\Delta_{\Phi_{\alpha}, \Psi_{\alpha}}\right)^{1 / 2}\right]^{-1}\right\| \leqq 1
$$

is uniformly bounded, we obtain

$$
\lim \left[\mathbb{1}+\left(\Delta_{\Phi_{\alpha}, \Psi_{\alpha}}\right)^{1 / 2}\right]^{-1} s^{M^{\prime}}(\Psi)=\left[\mathbb{1}+\left(\Delta_{\mathscr{\Phi}, \Psi}\right)^{1 / 2}\right]^{-1} s^{M^{\prime}}(\Psi) .
$$

The rest of the proof is standard. For $r>0$ and $\Delta_{\alpha}=\Delta_{\alpha}^{*} \geqq 0$,

$$
\left(r+\Delta_{\alpha}\right)^{-1}=R_{r}\left(\Delta_{\alpha}\right)\left(\mathbf{1}+\Delta_{\alpha}\right)^{-1}
$$

with

$$
\begin{aligned}
& R_{r}\left(\Delta_{a}\right)=\left\{\mathbb{1}+(r-1)\left(\mathbb{1}+\Delta_{\alpha}\right)^{-1}\right\}^{-1}, \\
& \left\|R_{r}\left(\Delta_{\alpha}\right)\right\| \leqq \max \left\{1, r^{-1}\right\} .
\end{aligned}
$$

If $\Delta=\Delta^{*} \geqq 0, \lim \left(\mathbf{1}+\Delta_{\alpha}\right)^{-1} s=(\mathbf{1}+\Delta)^{-1} s$ for a projection $s$ commuting with $\Delta$, then the formula

$$
\begin{aligned}
& \left(r+\Delta_{a}\right)^{-1}-(r+\Delta)^{-1}=R_{r}\left(\Delta_{\alpha}\right)\left\{\left(\mathbb{1}+\Delta_{\alpha}\right)^{-1}-(\mathbb{1}+\Delta)^{-1}\right\} \\
& \quad-R_{r}\left(\Delta_{\alpha}\right)(r-1)\left\{\left(\mathbb{1}+\Delta_{\alpha}\right)^{-1}-(\mathbb{1}+\Delta)^{-1}\right\} R_{r}(\Delta)(\mathbb{1}+\Delta)^{-1}
\end{aligned}
$$


implies

$$
\lim \left\{\left(r+\Delta_{\alpha}\right)^{-1}-(r+\Delta)^{-1}\right\} s=0,
$$

where the convergence is uniform if $r$ is restricted to any compact subset of $(0, \infty)$. By applying this result to $\Delta_{\alpha}=\left(\Delta_{\varpi_{\alpha}, \Psi_{\alpha}}\right)^{1 / 2}, \Delta=\left(\Delta_{\varpi, \Psi}\right)^{1 / 2}$ and $s=s^{M^{\prime}}(\Psi)$, we obtain the Lemma.

Q.E.D.

Proof of Theorem 3. 7 (1). We divide our proof into several steps. Obviously we may omit those $\alpha$ for which $s\left(\psi_{\alpha}\right) \geqq s\left(\phi_{\alpha}\right)$ does not hold out of our consideration so that we may assume $s\left(\psi_{\alpha}\right) \geqq s\left(\phi_{\alpha}\right)$ for all $\alpha$ without loss of generality.

(a) The case where $\psi$ is faithful: Due to $s(\psi)=\mathbb{1}$, we have $s^{M^{\prime}}(\Psi)=J s^{M}(\Psi) J=1$. Hence (4.2) and Lemma 4.1 imply

$$
\begin{aligned}
\lim \int_{\varepsilon}^{L} \mathrm{~d} r & \left(\Phi_{\alpha},\left\{(\mathbb{1}+r)^{-1}-\left[r+\left(\Delta_{\Phi_{\alpha}, \Psi_{\alpha}}\right)^{1 / 2}\right]^{-1}\right\} \Phi_{\alpha}\right) \\
& \left.=\int_{\varepsilon}^{L} \mathrm{~d} r\left(\Phi,\left\{(1+r)^{-1}-\left[r+(\lrcorner_{\varpi, \Psi}\right)^{1 / 2}\right]^{-1}\right\} \Phi\right)
\end{aligned}
$$

for all $0<\varepsilon<L<\infty$. (Note that

$$
\left\|\left[r+\left(\Delta_{\oplus_{\alpha} \cdot \Psi_{\alpha}}\right)^{1 / 2}\right]^{-1}\right\| \leqq r^{-1}
$$

is uniformly bounded.)

We also have the following estimates:

$$
\begin{aligned}
& \int_{0}^{\varepsilon} \mathrm{d} r\left|\left(\Phi_{\alpha},\left\{(1+r)^{-1}-\left[r+\left(\Delta_{\Phi_{\alpha}, \Psi_{\alpha}}\right)^{1 / 2}\right]^{-1}\right\} \Phi_{\alpha}\right)\right| \\
& \quad=\int_{0}^{\varepsilon} \mathrm{d} r\left|\int_{0}^{\infty}(1+r)^{-1}\left(1+r \lambda^{-1 / 2}\right)^{-1}\left(1-\lambda^{-1 / 2}\right) \mathrm{d}\left(\Phi_{\alpha}, E_{\lambda}^{\Phi_{\alpha}, \Psi_{\alpha}} \Phi_{\alpha}\right)\right| \\
& \quad \leqq \int_{0}^{\varepsilon} \mathrm{d} r \int_{0}^{\infty} \max \left(1, \lambda^{-1}\right) \mathrm{d}\left(\Phi_{\alpha}, E_{\lambda}^{\Phi_{\alpha}, \Psi_{\alpha}} \Phi_{\alpha}\right) \\
& \quad \leqq \varepsilon\left(\phi_{\alpha}(\mathbf{1})+\psi_{\alpha}(\mathbf{1})\right)
\end{aligned}
$$

due to (3.2), where $E_{\lambda}^{\Phi_{\alpha}, \Psi_{\alpha}}$ is the spectral projection of $\lrcorner_{\oplus_{\alpha}, \Psi_{\alpha}}$.

$$
\begin{aligned}
& \int_{L}^{\infty} \mathrm{d} r\left|\int_{0}^{1}\left\{(1+r)^{-1}-\left(r+\lambda^{1 / 2}\right)^{-1}\right\} \mathrm{d}\left(\Phi_{\alpha}, E_{\lambda}^{\Phi_{\alpha}, \Psi_{\alpha}} \Phi_{\alpha}\right)\right| \\
& \quad=\int_{L}^{\infty} \mathrm{d} r \int_{0}^{1}(1+r)^{-1}\left(1+r \lambda^{-1 / 2}\right)^{-1}\left(\lambda^{-1 / 2}-1\right) \mathrm{d}\left(\Phi_{\alpha}, E_{\lambda}^{{ }^{\omega_{\alpha}}, \Psi_{\alpha}} \Phi_{\alpha}\right)
\end{aligned}
$$




$$
\begin{aligned}
& \leqq \int_{L}^{\infty} \mathrm{d} r(1+r)^{-1} r^{-1} \int_{0}^{1} \mathrm{~d}\left(\Phi_{a}, E_{\lambda}^{\Phi_{a}, T_{a}} \Phi_{a}\right) \\
& \leqq \log \left(1+L^{-1}\right) \phi_{a}(\mathbb{1}) .
\end{aligned}
$$

Finally

$$
\int_{L}^{\infty} \mathrm{d} r \int_{1}^{\infty}\left\{(1+r)^{1}-\left(r+\lambda^{1 / 2}\right)^{-1}\right\} \mathrm{d}\left(\Phi_{a}, E_{\lambda}^{\Phi_{a}{ }^{y} a} \Phi_{a}\right) \geqq 0
$$

Hence

(4. 9) $\left.\liminf \int_{0}^{\infty} \mathrm{d} r\left(\Phi_{\alpha},\left\{(1+r)^{-1}-\left[r+(\lrcorner_{\omega_{a}, r_{a}}\right)^{1 / 2}\right]^{-1}\right\} \Phi_{a}\right)$

$$
\begin{array}{rl}
\geqq \int_{\varepsilon}^{L} & \mathrm{~d} r\left(\Phi,\left\{(1+r)^{-1}-\left[r+\left(A_{\tilde{\omega}, \Psi}\right)^{1 / 2}\right]^{-1}\right\} \Phi\right) \\
& -\varepsilon(\phi(\mathbb{1})+\psi(\mathbb{1}))-\left\{\log \left(1+L^{-1}\right)\right\} \phi(\mathbb{1}) .
\end{array}
$$

We now use the following formula, which holds if $s(\psi) \geqq s(\phi)$.

(4. 10) $S(\psi / \phi)=2 \int_{0}^{\infty}\left(\int_{0}^{\infty}\left\{(1+r)^{-1}-\left(r+\lambda^{1 / 2}\right)^{-1}\right\} \mathrm{d} r\right) \mathrm{d}\left(\Phi, E_{\lambda}^{\Phi, \psi} \Phi\right)$

$$
=2 \int_{0}^{\omega} \mathrm{d} r\left(\Phi,\left\{(1+r)^{-1}-\left[r+\left(d_{\Phi, \Psi}\right)^{1,2}\right] \quad\right\} \Phi\right),
$$

where the change of the order of $r$-and $\lambda$-integrations is allowed because the integral is definite in the Lebesgue sense (finite or $+\infty$ ) due to (3. 2).

By taking the limit $\varepsilon \rightarrow+0$ and $L \rightarrow+\infty$ and by substituting (4.10) and the same formula for the pair $\psi_{a}, \phi_{a}$, we obtain Theorem 3.7 (1) for this case.

(b) The case where $\phi_{a}$ is independent of $\alpha$ : By (3.3) and by the same computation as (4.10), we obtain

$$
S\left(\psi_{a} / \phi\right)=-2 \int_{0}^{\infty} \mathrm{d} r\left(\Phi,\left\{(1+r)^{-1}-\left[r+\left(d_{r_{a}, \emptyset}\right)^{1 / 2}\right]^{-1}\right\} \Phi\right)
$$

where the boundedness

$$
\int_{0}^{\infty} \lambda \mathrm{d}\left(\Phi, E_{\lambda}^{\prime \prime a, \Phi} \Phi\right)=\left\|\left(d_{Y_{a}, \Phi}\right)^{1 / 2} \Phi\right\|^{2}=\psi_{\alpha}(s(\phi))<\infty
$$

guarantees the definiteness of the integral in (4.11). (Note that $s\left(\psi_{\alpha}\right)$ $\geqq s\left(\phi_{\alpha}\right)=s(\phi)$.) 
By Lemma 4.1 and by the same argument as the Case (a), we obtain

(4.13) $\quad \lim \inf S\left(\psi_{\alpha} / \phi\right)$

$$
\geqq-2 \int_{0}^{\infty} \mathrm{d} r\left(\Phi,\left\{(1+r)^{-1}-\left[\mathrm{r}+\left(\Delta_{\Psi, \Phi}\right)^{1 / 2}\right]^{-1}\right\} \Phi\right) .
$$

Since $\left(\Delta_{\Psi, \oplus}\right)^{1 / 2}$ commutes with $s^{M}(\Psi)=J s^{M^{\prime}}(\Psi) J$, the inner product in (4.13) is the sum of contributions from the expectation values in ( 1 $\left.s^{M}(\Psi)\right) \Phi$ and $s^{M}(\Psi) \Phi$. The first one is given by

$$
-2 \int_{0}^{\infty} \mathrm{d} r\left(\Phi,\left\{(1+r)^{-1}-r^{-1}\right\}\left(\mathbb{1}-s^{M}(\Psi)\right) \Phi\right)=+\infty
$$

if

$$
\left(\Phi,\left(\mathbf{1}-s^{M}(\Psi)\right) \Phi\right)=\phi(\{\mathbf{1}-s(\psi)\})>0,
$$

i.e. if $s(\psi) \geqq s(\phi)$ does not hold. The second one is either finite or $+\infty$ by (4.12). Hence if $s(\psi) \geqq s(\phi)$ does not hold, then

$$
\lim S\left(\psi_{\alpha} / \phi\right)=+\infty=S(\psi / \phi) .
$$

If $s(\psi) \geqq s(\phi)$ holds, then (4.13) already proves Theorem 3.7 (1) for the present case.

(c) General case: Let $\omega$ be a normal faithful state. For $\varepsilon>0$, we obtain

$$
\lim \inf S\left(\psi_{\alpha}+\varepsilon \omega / \phi_{\alpha}\right) \geqq S(\psi+\varepsilon \omega / \phi)
$$

by the Case (a). By Theorem 3.6 (4),

$$
S\left(\psi_{\alpha}+\varepsilon \omega / \phi_{\alpha}\right) \leqq S\left(\psi_{\alpha} / \phi_{\alpha}\right) .
$$

Hence

$$
\lim \inf S\left(\psi_{a} / \phi_{\alpha}\right) \geqq S(\psi+\varepsilon \omega / \phi) .
$$

By taking the limit $\varepsilon \rightarrow+0$ and using the Case (b), we obtain Theorem 3.7 (1) for the general case.

Proof of Theorem 3.7 (2). If $\omega^{\prime} \geqq \lambda^{-1} \omega$ for $\lambda>0$, then (3.7) implies

$$
\left(\Delta_{\Omega^{\prime}, \Omega}+r\right)^{-1} \leqq\left(\lambda^{-1} \Delta_{\Omega, \Omega}+r\right)^{-1}
$$


Due to the identity

$$
\left(r+\rho^{1 / 2}\right)^{-1}=\pi^{-1} \int_{0}^{\infty}(\rho+x)^{-1}\left(x+r^{2}\right)^{-1} x^{1 / 2} d x, \quad r>0,
$$

for a positive self-adjoint $\rho$, this implies

$$
\left(r+\left(\Delta_{\Omega^{\prime}, \Omega}\right)^{1 / 2}\right)^{-1} \leqq\left(r+\lambda^{-1 / 2}\left(\Delta_{\Omega, \Omega}\right)^{1 / 2}\right)^{-1} .
$$

Hence

$$
\begin{aligned}
\omega(\mathbb{1}) & \geqq\left(\Omega,\left\{(1+r)^{-1}-\left[r+\left(\Delta_{\Omega^{\prime}, \Omega}\right)^{1 / 2}\right]^{-1}\right\} \Omega\right) \\
& \geqq \omega(\mathbb{1})\left\{(1+r)^{-1}-\left(\lambda^{-1 / 2}+r\right)^{-1}\right\} \\
& =\omega(\mathbb{1})(1+r)^{-1}\left(1+\lambda^{1 / 2} r\right)^{-1}\left(1-\lambda^{1 / 2}\right) .
\end{aligned}
$$

Therefore

$$
\begin{aligned}
-\varepsilon \omega(\mathbb{1}) & \leqq-\int_{0}^{\varepsilon} \mathrm{d} r\left(\Omega,\left\{(1+r)^{-1}-\left[r+\left(\Delta_{\Omega^{\prime}, \Omega}\right)^{1 / 2}\right]^{-1}\right\} \Omega\right) \\
& \leqq \omega(\mathbb{1}) \log \left\{(1+\varepsilon)\left(1+\lambda^{1 / 2} \varepsilon\right)^{-1}\right\}
\end{aligned}
$$

for $\varepsilon>0$. We also have for $L>0$

$$
\text { (4.16) } \begin{aligned}
\omega(\mathbb{1}) & \log \left(1+L^{-1}\right)=-\int_{L}^{\infty}\left(\Omega,\left\{(1+r)^{-1}-r^{-1}\right\} \Omega\right) \\
& \geqq-\int_{L}^{\infty} \mathrm{d} r\left(\Omega,\left\{(1+r)^{-1}-\left[r+\left(\Delta_{\Omega^{\prime}, \Omega}\right)^{1 / 2}\right]^{-1}\right\} \Omega\right) \\
& =-\int_{L}^{\infty} \mathrm{d} r\left(\Omega,(1+r)^{-1}\left[r+\left(\Delta_{\Omega^{\prime}, \Omega}\right)^{1 / 2}\right]^{-1}\left\{\left(\Delta_{\Omega^{\prime}, \Omega}\right)^{1 / 2}-\mathbf{1}\right\} \Omega\right) \\
& \geq-\int_{L}^{\infty}(1+r)^{-1} r^{-1} \mathrm{~d} r\left\|\left(\Delta_{\Omega^{\prime}, \Omega}\right)^{1 / 2} \Omega\right\|^{2} \\
& =-\omega^{\prime}(\mathbf{1}) \log \left(1+L^{-1}\right) .
\end{aligned}
$$

where the last inequality is obtained by using the spectral decomposition $\Delta_{\Omega^{\prime}, \Omega}=\int \lambda \mathrm{d} E_{\lambda}$ and majorizing $\left(r+\lambda^{1 / 2}\right)^{-1}\left(\lambda^{1 / 2}-1\right)$ by $r^{-1} \lambda$ for $0 \leqq \lambda$. Since

$$
\begin{aligned}
\lim \int_{\varepsilon}^{L} \mathrm{~d} r\left(\Phi_{a},\left\{(1+r)^{-1}-\left[r+\left(\Delta_{\Psi_{\alpha}, \Phi_{\alpha}}\right)^{1 / 2}\right]^{-1}\right\} \Phi_{\alpha}\right) \\
\quad=\int_{\varepsilon}^{L} \mathrm{~d} r\left(\Phi,\left\{(1+r)^{-1}-\left[r+\left(\Delta_{T, \oplus}\right)^{1 / 2}\right]^{-1}\right\} \Phi\right),
\end{aligned}
$$

the estimates (4.15) and (4.16) for $\left(\omega^{\prime}, \omega\right)=\left(\psi_{\alpha}, \phi_{\alpha}\right)$ and for $\left(\omega^{\prime}, \omega\right)$ $=(\psi, \phi)$ yield 


$$
\begin{aligned}
\lim \int_{0}^{\infty} \mathrm{d} r\left(\Phi_{\alpha},\left\{(1+r)^{-1}-\left[r+\left(\Delta_{\Psi_{\alpha}, \Phi_{\alpha}}\right)^{1 / 2}\right]^{-1}\right\} \Phi_{\alpha}\right) \\
\quad=\int_{0}^{\infty} \mathrm{d} r\left(\Phi,\left\{(1+r)^{-1}\left[r+\left(\Delta_{\Psi, \Phi}\right)^{1 / 2}\right]^{-1}\right\} \Phi\right)
\end{aligned}
$$

Since $\lambda \psi_{\alpha} \geqq \phi_{\alpha}$ and its consequence $\lambda \psi \geqq \phi$ imply $s\left(\psi_{\alpha}\right) \geqq s\left(\phi_{\alpha}\right)$ and $s(\psi)$ $\geqq s(\phi)$, the equations (4.17) and an expression of the form (4.11) for $s\left(\psi_{\alpha} / \phi_{a}\right)$ and $s(\psi / \phi)$ imply Theorem 3.7 (2).

Proof of Theorem 3.7 (3). This follows from Theorem 3.7 (1) and Theorem 3.6 (4).

Remark 4.2. The argument leading to (4.14) implies that the formula

$$
\text { (4. 18) } S(\psi / \phi)=-2 \int_{0}^{\infty} \mathrm{d} r\left(\Phi,\left\{(1+r)^{-1}-\left[r+\left(\Delta_{\Psi, \oplus}\right)^{1 / 2}\right]^{-1}\right\} \Phi\right),
$$

which is used in (4.11) for the case $s(\psi) \geqq s(\phi)$, holds for a general pair $\psi$ and $\phi$ (even if $s(\psi) \geqq s(\phi)$ does not hold). this is not the case for the formula of the form (4.10).

Proof of Theorem 3.9 (1). Let $\omega_{0}$ be a faithful state, $\omega=\omega_{0}+\psi+\phi$, and $1>\varepsilon>\| 1>0$. The proof of Lemma 3 in [1] (without the assumption $\psi \leqq k \phi$ there) implies

$$
\lim \inf S\left(E_{N_{\alpha}} \psi_{\varepsilon} / E_{N_{\alpha}} \phi_{\mu}\right) \geqq S\left(\psi_{\varepsilon} / \phi_{\mu}\right)
$$

where

$$
\psi_{\varepsilon}=(1-\varepsilon) \psi+\varepsilon \omega, \quad \phi_{\mu}=(1-\mu) \phi+\mu \omega .
$$

By the convexity (Theorem 3.8 (1)),

$$
\begin{aligned}
& S\left(E_{N_{\alpha}} \psi_{\varepsilon} / E_{N_{\alpha}} \phi_{\mu}\right) \\
& \quad \leqq(1-\mu) S\left(E_{N_{\alpha}} \psi_{\varepsilon} / E_{N_{\alpha}} \phi\right)+\mu S\left(E_{N_{\alpha}} \psi_{\varepsilon} / E_{N_{\alpha}} \omega\right) .
\end{aligned}
$$

By Theorem 3.6 (4) and (3), we have

$$
\begin{gathered}
S\left(E_{N_{\alpha}} \psi_{\varepsilon} / E_{N_{\alpha}} \omega\right) \leqq S\left(E_{N_{\alpha}}(\varepsilon \omega) / E_{N_{\alpha}}(\omega)\right. \\
=-\omega(\mathbb{1}) \log \varepsilon<\infty .
\end{gathered}
$$

By Theorem 3.7 (2) 
The formulas (4.20), (4.21) and (4.22) imply in the limit $\mu \rightarrow+0$

By Theorem 3.7 (3),

$$
\lim _{\varepsilon \rightarrow \pi} S\left(\psi_{\varepsilon} / \phi\right)=S(\psi / \phi)
$$

By Theorem 3.6 (4),

$$
\begin{array}{r}
S\left(E_{N_{\alpha}} \psi_{\varepsilon} / E_{N_{\alpha}} \phi\right) \leqq S\left(E_{N_{\alpha}}(1-\varepsilon) \psi / E_{N_{\alpha}} \phi\right) \\
=S\left(E_{N_{\alpha}} \psi / E_{N_{\alpha} \alpha} \phi\right)-\phi(\mathbf{1}) \log (1-\varepsilon) .
\end{array}
$$

The formulas (4.23), (4.24) and (4.25) imply in the limit $\varepsilon \rightarrow+0$ Theorem 3.9 (1).

Proof of Theorem 3.9 (2). This follows from Theorem 3.9 (1) and Theorem $3.8(2)(\gamma)$.

Q.E.D.

Proof of Theorem 3. 10. First consider the case where $\phi$ is faithful. Then $\Omega$ given by (2.8) is cyclic and separating for $M$. From the definition of the perturbed state and the expression (2.10), we obtain

$$
\Psi(h) \otimes e_{11}+\Phi \otimes e_{22}=\Omega\left(h \otimes u_{11}\right) .
$$

By (4.13) of [8], we have

$$
\left.\log \lrcorner_{\Omega\left(h \otimes u_{11}\right)}=\log \right\lrcorner_{\Omega}+h \otimes u_{11}-j(h) \otimes J_{K} u_{11} J_{k} .
$$

Here $j(h)$ denotes $J h J$. By (2.10), we obtain

$$
\begin{aligned}
& \log \Delta_{\Psi(h), \emptyset}=\log \Delta_{\Psi, \downarrow}+h, \\
& \log \Delta_{\emptyset . \downarrow(h)}=\log \Delta_{\varpi, \Psi}-j(h) .
\end{aligned}
$$

By (3. 3), for example, we obtain Theorem 3. 10 for the present case of a faithful $\phi$.

For the general case, we apply the result just proved to

$$
\phi_{s}=(1-\varepsilon) \phi+\varepsilon \psi, \quad \varepsilon>0,
$$

which is faithful: 


$$
S\left(\psi^{h} / \phi_{\varepsilon}\right)=-(1-\varepsilon) \phi(h)-\varepsilon \psi(h)+S\left(\psi / \phi_{\varepsilon}\right) .
$$

From the convexity of the relative entropy, we obtain

$$
S\left(\psi^{h} / \phi_{\varepsilon}\right) \leqq(1-\varepsilon) S\left(\psi^{h} / \phi\right)-\varepsilon \psi(h) .
$$

Combining the limit $\varepsilon \rightarrow+0$ of this relation with Theorem 3.7 (1), we obtain

$$
\lim _{\varepsilon \rightarrow+0} S\left(\psi^{h} / \phi_{\varepsilon}\right)=S\left(\psi^{h} / \phi\right)
$$

For $h=0$, we have the same equation for $\phi$. Hence the first equation of Theorem 3.10 follows from (4.30). The second equation of Theorem 3.10 is trivially true for a non-faithful $\phi$ because both sides of the equation is then $+\infty$.

\section{$\S 5$. Relative Entropy of States of $C^{*}$-Algebras}

For two positive linear functionals $\psi$ and $\phi$ of a $C^{*}$-algebra $\mathfrak{A}$, we define the relative entropy $S(\psi / \phi)$ by

$$
S(\psi / \phi) \equiv S(\widetilde{\psi} / \widetilde{\phi})
$$

where $\widetilde{\psi}$ and $\widetilde{\phi}$ are the unique normal extension of $\psi$ and $\phi$ to the enveloping von Neumann algebra $\mathfrak{X}$ '”.

If the cyclic representation $\pi_{\psi}$ associated with $\psi$ does not quasi-contain the cyclic representation $\pi_{\phi}$ associated with $\phi$, then the central support of $\widetilde{\psi}$ does not majorize that of $\widetilde{\phi}$, hence $s(\widetilde{\psi}) \geq s(\widetilde{\phi})$ does not hold. Therefore

$$
S(\psi / \phi)=+\infty
$$

if $\pi_{\psi}$ does not quasi-contain $\pi_{\phi}$.

From the definition (5.1), it follows that

$$
S(\psi / \phi)=S(\widehat{\psi} / \widehat{\phi})
$$

where $\hat{\psi}$ and $\hat{\phi}$ are the unique normal extension of $\psi$ and $\phi$ to $M=\pi(\mathfrak{U})$ " where $\pi=\pi_{\psi} \oplus \pi_{\phi}$. If $\mathfrak{A}$ is separable, then $M=\pi(\mathfrak{H})^{\prime \prime}$ for this $\pi$ has a separable predual and hence all results in previous sections apply. In particular, if $\mathfrak{U}_{\alpha}$ is a monotone increasing net of nuclear $C^{*}$-subalgebras of $\mathfrak{l}$ generating $\mathfrak{A}$, then 


$$
\lim S\left(E_{\mathfrak{A}_{\alpha}} \psi / E_{\mathfrak{A}_{\alpha}} \phi\right)=S(\psi / \phi) .
$$

This implies the result in [2] that if

$$
\sup _{a} S\left(E_{\mathfrak{A}_{\alpha}} \psi / E_{\mathfrak{A}_{\alpha}} \phi\right)<\infty \text {, }
$$

then $\pi_{\psi}$ quasi-contains $\pi_{\phi}$.

If $\mathfrak{i l}$ is separable, then the restriction of the envelopping von Neumann algebra $\mathfrak{U}^{\prime \prime}$ to a direct sum of a denumerable number of cyclic representations of $\mathfrak{i}$ has a faithful normal state. Hence Theorems 3.6, 3. 8, and 3. 9 as well as Theorem 3.7 for sequences are valid for positive linear functionals of $C^{*}$-algebras.

If $\psi$ is a positive linear functional of a $C^{*}$-algebra $\mathfrak{i}$ such that the corresponding cyclic vector $\Psi$ for the associated cyclic representation $\pi_{\psi}$ of $\mathfrak{A}$ is separating for the weak closure $\left.\pi_{\psi}(\mathfrak{A})\right)^{\prime \prime}$, then the perturbed state $\psi^{h}$ for $h=h^{*} \in \mathfrak{Q}$ is defined by

$$
\psi^{h}(a)=\left(\Psi\left[\pi_{\psi}(h)\right], \pi_{\psi}(a) \Psi\left[\pi_{\psi}(h)\right]\right), \quad a \in \mathfrak{H} .
$$

For such $\psi$, Theorem 3. 10 holds for $C^{*}$-algebras.

\section{§6. Conditional Entropy}

Let $\mathfrak{U}$ be a UHF algebra with an increasing suquence of finite dimensional factors $\mathfrak{H}_{n}$ generating $\mathfrak{U}$. Let $\mathfrak{U}_{m, n}^{c}$ be the relative commutant of $\mathfrak{U}_{n}$ in $\mathfrak{U}_{m}$. The conditional entropy $\widetilde{S}_{n}(\phi)$ of a positive linear functional $\phi$ of $\mathfrak{P}$ is defined by

$$
\widetilde{S}_{n}(\phi)=\lim _{m \rightarrow \infty}\left(S\left(E_{\mathfrak{A}_{m}} \phi\right)-S\left(E_{\mathfrak{A}_{m, n}^{\mathrm{c}}} \phi\right)\right)
$$

where

$$
S(\psi)=-\psi\left(\log \rho_{\psi}\right)
$$

for a positive linear functional $\psi$ of a finite dimensional factor $\mathfrak{M}_{k}$ and $\rho_{\psi}$ is the density matrix of $\psi$ defined by

$$
\psi(a)=\tau\left(\rho_{\psi} a\right), \quad a \in \mathfrak{M}_{k}
$$

with the unique trace state $\tau$ of $\mathfrak{M}_{k}$. ([3])

Let $\mathfrak{U}^{\mathrm{c}}{ }_{n}$ be the relative commutant of $\mathfrak{U}_{n}$ in $\mathfrak{H}, \omega$ be the restriction of $\phi$ to $\mathfrak{A l}^{c}{ }_{n}$ and $\omega^{\prime}$ be any positive linear functional on $\mathfrak{U}^{c}{ }_{n}$. Then 
(6. 2) $\quad S\left(E_{\mathfrak{Q}_{n}} \phi\right)-S\left(E_{\mathfrak{A}_{m, n}^{c}}^{c} \phi\right)=-S\left(E_{\mathfrak{Q}_{m}}\left(\tau_{n} \otimes \omega^{\prime}\right) / E_{\mathfrak{Q}_{m}} \phi\right)+S\left(E_{\mathfrak{Q}_{m, n}^{c}}^{\mathrm{c}} \omega^{\prime} / E_{\mathfrak{Q}_{m, n}^{c}}^{c} \omega\right)$

where $\tau_{n}$ is the unique trace state on $\mathfrak{A}_{n}$, because the density matrices for $E_{\mathfrak{A}_{m}}\left(\tau_{n} \otimes \omega^{\prime}\right)$ and for $E_{\mathfrak{A}_{m, n}^{c}} \omega^{\prime}$ are the same element of $\mathfrak{A}$.

By taking the limit $m \rightarrow \infty$ and using (5.4), we obtain

$$
\left.\widetilde{S}_{n}(\phi)=S(\omega)^{\prime} /(1)\right)-S\left(\tau_{n} \otimes \omega^{\prime} / \phi\right) .
$$

Since the left hand side is finite, it follows that if either $S\left(\omega^{\prime} / \omega\right)$ or $S\left(\tau_{n} \otimes \omega^{\prime} / \phi\right)$ is finite, then both quantities are finite and (6.3) holds. This formula has been used in [3].

\section{References}

[1] Araki, H., Publ. RIMS Kyoto Univ. 11 (1975-76), 809-833.

[2] - Commun. Math. Phys., 44 (1975), 1-7.

[3] and Sewell, G. L., Commun. Math. Phys., 52 (1977), 103-109.

[4] - Pacific J. Math., 50 (1974), 309-354.

[5] Connes, A., Ann. Inst. Fourier, Grenoble., 24, 4 (1974), 121-155.

[6] Haagerup, U., Math. Scand., 37 (1975), 271-283.

[7] Connes, A., Ann. Scient. Ecole Norm. Sup. 4e série, 6 (1973), 133-252.

[8] Araki, H., Publ. RIMS Kyoto Univ., 9 (1973), 165-209. 